\title{
Chaotic Vibrations of a Clamped-Supported Beam with a Concentrated Mass Subjected to Static Axial Compression and Periodic Lateral Acceleration"
}

\author{
Dai YANAGISAWA ${ }^{* *}$, Ken-ichi NAGAI $^{* * *}$ and Shinichi MARUYAMA ${ }^{* * *}$ \\ ${ }^{* *}$ MITSUBA Corporation, \\ 1-2681 Hirosawa-cho, Kiryu, Gunma 376-8555, Japan \\ E-mail: d-yanagi@mitsuba.co.jp \\ ${ }^{* * *}$ Department of Mechanical System Engineering, graduate school of engineering Gunma University, \\ 1-5-1, Tenjin-cho, Kiryu, Gunma 376-8515, Japan
}

\begin{abstract}
Experimental results and analytical results are presented on chaotic vibrations of a clamped-supported beam with a concentrated mass. The beam is elastically compressed by an axial spring at the simply supported end and is excited by lateral periodic acceleration. In the experiment, periodic and chaotic vibrations are detected under several conditions of the axial compression. In the analysis, the governing equation is reduced to nonlinear differential equations of a multiple-degree-of-freedom system by the Galerkin procedure. The nonlinear periodic responses are calculated by the harmonic balance method. The chaotic responses are numerically integrated by the Runge-Kutta-Gill method. The chaotic responses of the beam are examined with the Fourier spectra, the Poincaré projections and the maximum Lyapunov exponents and the principal component analysis. Under a specific axial compression with post-buckled state of the beam, the chaotic vibrations dominated by dynamic snap-through are generated by the ultra-sub-harmonic resonance response of $2 / 3$ order of the fundamental vibration mode. The number of pre-dominant vibration modes that contribute to the chaos is found to be three. Decreasing the axial compression, the chaotic vibrations are induced by the internal resonance response between the second and the fundamental mode of vibration. The number of predominant vibration modes that contribute to the chaos is found to be two or three. Both results of the experimental and the analysis agree remarkably with each other in detail.
\end{abstract}

Key words: Nonlinear Vibration, Vibration of Continuous System, Chaos and Fractal, Poincaré Projection, Lyapunov Exponent, PCA

\section{Introduction}

Thin walled structural elements such as beams and arches are used in many vehicles. The beams are usually connected to other elastic elements at their boundaries in the structures. The beam is subjected to the axial compression, when axial displacement acts on the beam trough the elastic elements. Under the axial compression, buckling of the beam is induced at a critical compressive force. As the post-buckled beam has a sufficient curvature, the beam has higher bending rigidity than that of a straight beam. However, snap-through buckling of the post-buckled beam occurs under critical lateral force. Under periodic lateral force, chaotic vibrations are generated in typical frequency ranges of the periodic force. The 
chaotic vibrations are sensitive to a slight variation of the initial deflection and static deformation of the beam. Furthermore, since lateral deflection of the beam has nonlinear coupling with axial displacement, the generation of the chaos is drastically influenced by the small fluctuations of the axial elastic constraint. The chaotic response of the beam shows a violent movement due to dynamic snap-through transitions. Therefore, multiple modes of vibration are generated simultaneously in the chaotic response.

The authors have investigated precisely the chaotic vibrations dominated by dynamic snap-through both experimentally and analytically ${ }^{(1-4)}$. For the both ends clamped beam, the main chaotic responses of the post-buckled beam were dominated by the sub-harmonic resonance responses of $1 / 2$ order and $1 / 3$ order with the fundamental mode of vibration ${ }^{(1-3)}$. Asymmetric modes of vibration were prominently induced in the chaotic vibrations of a beam which has a concentrated mass at asymmetric position of the beam ${ }^{(2)}$. For the clamped-supported beam ${ }^{(4)}$, predominant chaotic responses were dominated by the sub-harmonic resonance response of $1 / 2$ order and the ultra-sub-harmonic resonance response of $2 / 3$ order of the fundamental mode of vibration, simultaneously.

In this paper, to reveal the features of chaotic vibrations of the clamped-supported beam with a concentrated mass under the static axial compression, experimental and analytical results are presented precisely. In the experiment, a concentrated mass is attached at an asymmetric position of the beam to induce asymmetric modes of vibration. When the beam is loaded by an axial compression until the post-buckled state, the beam has linear natural frequencies $f_{2}$ and $f_{1}$ hold the nearly one-to-two ratio under a certain particular axial compression. Decreasing the axial compression, chaotic vibrations dominated with dynamic snap-through are generated within wide frequency range at a particular axial compressive force. Furthermore, decreasing the axial compression, chaotic vibrations concerning a natural frequency $f_{2}$ are induced within narrow frequency range. In the analysis, the chaotic responses are calculated to compare with the results of the experiment. Both results of the experiment and analysis remarkably coincide in detail.

\section{Test beam and fixture}

Fig.1 shows clamped-supported beam and its fixture. As shown in the figure, the coordinate system is denoted by $x$-axis and $z$-axis, where the direction of $z$-axis corresponds to the direction of gravity. A thin phosphor bronze beam with thickness $h=0.303 \mathrm{~mm}$, breadth $b=40.0 \mathrm{~mm}$ and length $188 \mathrm{~mm}$ is clamped at one end. The actual length of the beam is $L=140 \mathrm{~mm}$. To detect lateral displacement of the beam by a laser displacement sensor, both surfaces of the beam are painted white as a reflection target. Material properties of the beam are measured as the Young's modulus $E=118 \mathrm{GPa}$ and the mean mass density $\rho=8.88 \times 10^{3} \mathrm{~kg} / \mathrm{m}^{3}$. To minimize deflection and bending rigidity at simply supported end, the beam is connected to an elastic plate with thickness $0.80 \mathrm{~mm}$ by strips of adhesive films. The elastic plate is clamped by a slide block and works as the axial spring. Consequently, the beam has the clamped-supported boundary condition in the lateral direction and is elastically constrained in the axial direction. Seven couples of magnets $\left(m=0.43 \times 10^{-3} \mathrm{~kg}\right)$ are attached to the beam at $x=42 \mathrm{~mm}$. Then, the total weight as a concentrated mass is $M=6.1 \times 10^{-3} \mathrm{~kg}$. When the slide block on the base plate is compressed inward, the compressive axial force is loaded on the beam. The lowest natural frequency of the elastic plate without the beam is $191 \mathrm{~Hz}$ and is 4.7 times of the lowest natural frequency of the beam.

\section{Vibration test apparatus}

The beam is excited under periodic acceleration with a system of an electromagnetic exciter through the fixture. A schematic diagram of the test apparatus is shown in Fig.2. In the figure, devices numbered from 1 to 4 compose the set of the electromagnetic exciter. The exciter controller 1 generates a periodic sinusoidal signal which is amplified by the 
power amplifier 2 . The vibration exciter 3 drives the fixture with periodic acceleration. The signal of acceleration detected by the acceleration pick-up 4 . The signal is fed back to the exciter controller 1. Then, the peak acceleration is kept at a prescribed constant level. The instruments of laser displacement sensors are numbered from 6 to 7. The sensor 6 detects total displacements of the beam 5 at five positions along the beam, simultaneously. While, the sensor 7 measures the displacement of the base plate only. By differential operation of these signals, relative displacements of the beam to the displacement of the base plate are detected. The frequency counter 8 measures the frequency of the periodic excitation. The digital voltmeter 9 transforms the response of the beam to the root mean square value. Then, frequency response curves of the beam are monitored by the computer 10. The multi-channel digital recorder 11 acquires the dynamic responses. Then, time histories of the dynamic responses are transferred to the computer 10. The computer 10 transforms the dynamic response to the Fourier spectrum. The Poincare projection of the chaotic response is recorded as follows: the displacement of the chaotic response is differentiated to the velocity by the differential amplifier 13 . The phase meter 14 detects the maximum point of the periodic acceleration of the excitation and then the pulse oscillator 15 sends a timing signal to the digital recorder 12 with a prescribed phase shift. Then, the recorder 12 captures both the aforementioned displacement and velocity of the chaotic response. Finally, computer 10 records the Poincaré projection of the chaos.

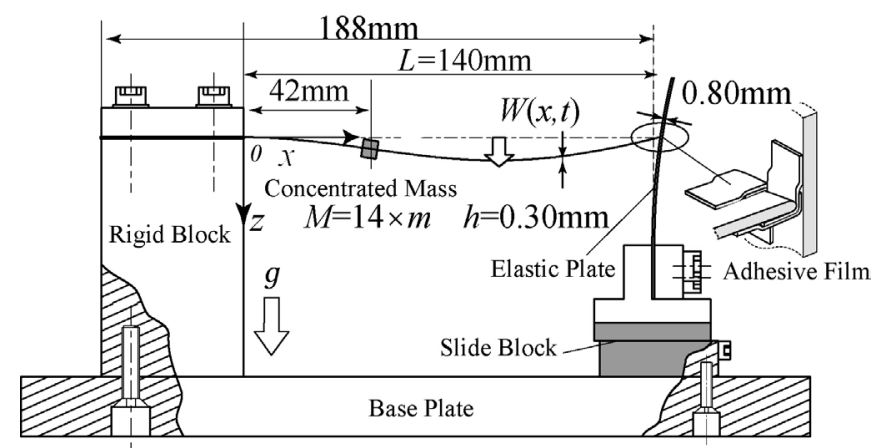

Fig. 1 Fixture of the beam with concentrated mass and the axial elastic constraint.

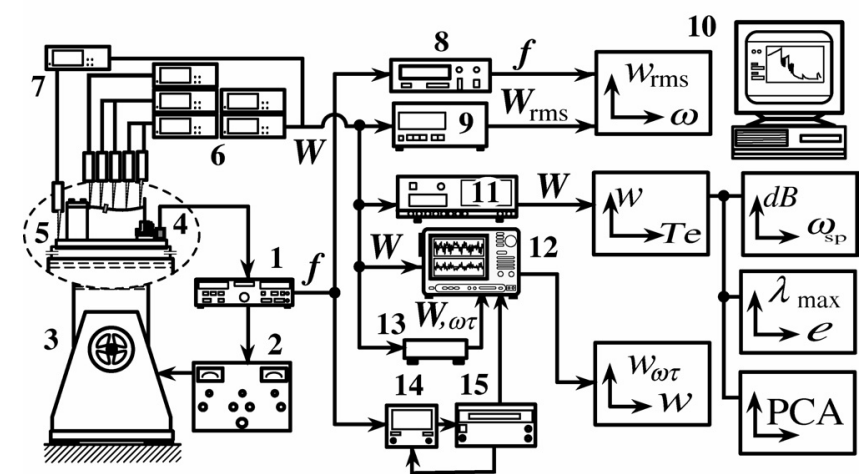

Fig. 2 Vibration test apparatus of the beam

\section{Test procedure}

First, the initial deflection is measured with a condition that breadth direction of the beam set to the direction of the gravity. Then, the lateral deflection by the gravity can be minimized. The initial deflection is approximated by the method of least squares.

To find fundamental properties of the beam, the linear natural frequencies and the restoring force are inspected. The beam is initially subjected to the static lateral force by the gravitational acceleration. The natural frequencies of the beam are measured by increasing the axial compressive force. To obtain the characteristics of restoring force, the relation 
between the static lateral deflection and a static concentrated force is measured with the laser displacement sensor and a load cell.

Next, by applying the periodic excitation on the beam, chaotic responses of the beam are investigated. The peak amplitude of periodic excitation is selected as $a_{d}=26 \mathrm{~m} / \mathrm{s}^{2}$. Chaotic vibrations are detected under several conditions of the axial compression. To find frequency regions where chaotic responses are generated, the nonlinear frequency response curve is inspected under the each condition. The exciting frequency is changed slowly within the range from $f=30 \mathrm{~Hz}$ to $f=100 \mathrm{~Hz}$ with the sweep rate of the frequency $0.1 \mathrm{~Hz} / \mathrm{s}$. Time responses of the chaotic vibrations are examined with the Fourier spectra, the Poincare projections and the maximum Lyapunov exponents. The maximum Lyapunov exponent $\lambda_{\max }$ of the chaotic response is calculated with the procedure by Wolf et al. ${ }^{(5)}$ and Takens $^{(6)}$. Increasing the embedding dimension $e$ in the pseudo phase space, if the maximum Lyapunov exponent $\lambda_{\max }$ is saturated to a constant value, the maximum Lyapunov exponent $\lambda_{\max }$ and the embedding dimension $e$ of the inspected response are determined. If the $\lambda_{\max }$ takes a positive value, the response is confirmed as the chaos. Half of the embedding dimension corresponds to the number of vibration modes that contribute predominantly to the chaotic response.

Furthermore, measuring simultaneous responses at multiple positions of the beam, the principal components are calculated by the Karhunen-Loéve transformation ${ }^{(7)}$. The K-L transformation enables one to estimate contribution ratio and related modal pattern in the chaotic response of the beam. Results of the principal component analysis presents an optimal estimation of linear modes to the nonlinear time responses ${ }^{(8)}$.

\section{Analytical procedure}

As shown in Fig.3, a thin beam with length $L$ is clamped and supported at the ends. The area of the cross section of the beam is $A=b h$. Notation $I$ stands for the moment of inertia of cross section. Simply supported end of the beam is connected to an axial spring with the spring coefficient $K$. The axial spring is compressed by an initial axial displacement $U_{0}$. Coordinate system of the beam is defined with $x$-axis along the beam and $z$-axis to lateral direction. The beam is subjected to the lateral acceleration $a=g+a_{d} \cos \Omega t$. Notations $\Omega$ and $t$ are the exciting angular frequency and time, respectively. Assuming the beam with lower bending vibration, effects of axial inertia, rotating inertia and shear deformation can be neglected. Notations $W_{0}(x), W(x, t)$ denote the initial deflection and the total deflection, respectively. The constant axial displacement $U_{0}$ is given to the outer end of the axial spring. The governing equation for the non-linear vibration of the beam is given with the non-dimensional notations

$$
\begin{aligned}
& L(\hat{w}) \equiv\left[1+\beta \delta\left(\xi-\xi_{1}\right)\right] \hat{w}_{\tau \tau}+\left(\hat{w}-w_{0}\right)_{{ }_{\xi \xi \xi \xi \xi}}-n_{x} \hat{w},_{\xi \xi} \\
& -\left[1+\beta \delta\left(\xi-\xi_{1}\right)\right]\left(p_{s}+p_{d} \cos \omega \tau\right)-\delta\left(\xi-\xi_{2}\right) q_{s}=0, \\
& n_{x}=\bar{k}\left[-u_{0}+\int_{0}^{1} \frac{1}{2}\left(\hat{w}_{, \xi}^{2}-w_{0}, 2\right) d \xi\right] \text {. }
\end{aligned}
$$

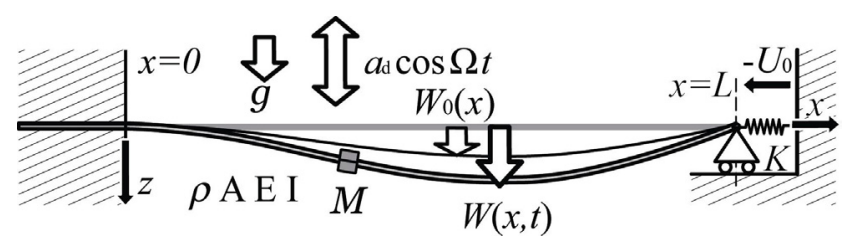

Fig. 3 Clamped-supported beam with a concentrated mass and co-ordinate system.

The equation includes the nonlinear effects of axial deformation due to large deflections of $\hat{w}$ and $w_{0}$. Subscript following a comma stands for partial differentiation. The Dirac's 
delta function is denoted by $\delta\left(\xi-\xi_{i}\right)(i=1,2)$. The following non-dimensional notations are introduced.

$$
\begin{aligned}
& {\left[\xi, \xi_{1}, \xi_{2}\right]=\left[x, x_{1}, x_{2}\right] / L,\left[\hat{w}, w_{0}\right]=\left[W, W_{0}\right] / r, \beta=M / \rho A L,} \\
& n_{x}=N_{x}\left(L^{2} / E I\right), u_{0}=\left(L / r^{2}\right) U_{0}, k=K L / E A, \bar{k}=\left(k^{-1}+1\right)^{-1}, \\
& {\left[p_{s}, p_{d}\right]=\left[g, a_{d}\right] \rho A L^{4} / E I r, q_{s}=Q_{s} L^{3} / E I r, \tau=\Omega_{0} t, \omega=\Omega / \Omega_{0}} \\
& r=\sqrt{I / A}, \Omega_{0}=\left(1 / L^{2}\right) \sqrt{E I / \rho A}
\end{aligned}
$$

In Eq.(3), $r$ is the radius of gyration of the cross section and $\Omega_{0}$ represents the coefficient of lateral vibration of the beam. In the notation in Eq.(2), $\xi$ is the non-dimensional coordinate, $\hat{w}$ and $w_{0}$ are the non-dimensional deflection normalized by the radius of gyration $r . \beta$ is ratio of a concentrated mass to the total mass of the beam. The notation $n_{x}$ and $u_{0}$ represent the non-dimensional quantity of the axial force $N_{x}$ acting on the cross section of the beam and initial axial displacement, respectively. $k$ is the coefficient of the axial spring normalized by the equivalent spring-coefficient of the beam in a longitudinal deformation. The notation $p_{s}$ and $p_{d}$ are the non-dimensional load intensities related to the gravity and the periodic excitation, respectively. When the characteristics of restoring force of the beam are examined, the deflection is calculated under the concentrated lateral force $Q_{s}$ applied on the beam. The notation $q_{s}$ is non-dimensional load intensity. For the beam that one end is clamped and the other is supported, the boundary conditions for the total deflection and the initial deflection are given as:

$$
\begin{aligned}
& \xi=0 ; \hat{w}=0, \hat{w}_{\xi \xi}=0, w_{0}=0, w_{0}, r_{\xi}=0, \\
& \xi=1 ; \hat{w}=0, \hat{w}_{\xi_{\xi}}=0, w_{0}=0, w_{0}, \xi_{\xi \xi}=0 .
\end{aligned}
$$

Using the Galerkin procedure, the governing equation is reduced to the equation of a multiple-degree-of-freedom system. The deflection $\hat{w}$ and $w_{0}$ are assumed as:

$$
\begin{aligned}
& {\left[\hat{w}, w_{0}\right]=\sum_{j}\left[\hat{b}_{j}(\tau), a_{j}\right] \zeta_{j}(\xi),(j=1,2,3, \cdots)} \\
& \zeta_{\mathrm{j}}(\xi)=e_{j}(\xi) f(\xi), \\
& e_{j}(\xi)=\cos (j-1) \pi \xi, f(\xi)=\sum_{k=1}^{5} c_{k} \xi^{k-1}
\end{aligned}
$$

where $\hat{b}_{j}(\tau)$ is the unknown time function and $a_{j}$ is the given constant to denote the initial deflection. $\zeta_{j}(\xi)$ is the mode shape function proposed by the senior author ${ }^{(9)}$. The mode shape function $\zeta_{j}(\xi)$ is expressed as the product of the trigonometric function $e_{j}(\xi)$ and the truncated power series $f(\xi)$. The function $e_{j}(\xi)$ enables the deflection $\hat{w}$ to include higher modes of vibration, while the power series $f(\xi)$ satisfies the boundary condition by choosing the coefficients $c_{k}$ appropriately. In this analysis of the clamped supported beam, the coefficients $c_{k}$ of the power series $f(\xi)$ are expressed as:

$$
c_{1}=0, c_{2}=0, c_{3}=3, c_{4}=-5, c_{5}=2
$$

Substituting Eqs. (5) into the governing equation of Eq. (1) and applying the Galerkin procedure, one obtain a set of nonlinear ordinary differential equations. To find the deflection of the beam by the static force, the terms of inertia force and the periodic force are neglected. The unknown time coefficients $\hat{b}_{j}$ are replaced to simultaneous cubic equations in terms of $\bar{b}_{j}$. Reducing the equations to simultaneous cubic equations in terms of $\bar{b}_{j}(j=1,2,3, \ldots)$, one obtains the static deflection and the static equilibrium by the substitution of $\bar{b}_{j}$ into Eq. (5). Introducing new coordinates $\tilde{b}_{j}(\tau)$ for displacement and putting $\hat{b}_{j}=\bar{b}_{j}+\tilde{b}_{j}(\tau)$, one can obtain the nonlinear equations with quadratic and cubic terms for the new coordinates $\tilde{b}_{j}$ of which origins are shifted to the static equilibrium position. Omitting the nonlinear terms and the periodic force, one obtains a set of 
homogeneous linear equations for free vibration of small amplitude. From the equation, both the natural frequencies $\omega_{i}$ and linear natural modes of vibration $\tilde{\zeta}_{i}(i=1,2,3, \ldots)$ are calculated. Transforming the equation of the variable $\tilde{b}_{i}(\tau)$ to the normal coordinates $b_{i}^{(p)}(\tau)(p=1,2)$ corresponding to $\tilde{\zeta}_{i}(\xi)$, a set of nonlinear differential equations in the state space of $b_{i}^{(p)}(\tau)(p=1,2: i=1,2,3, \ldots)$ is expressed as:

$$
\begin{aligned}
b_{i}^{(1)}{ }_{\tau}= & b_{i}^{(2)} \\
b_{i}^{(2)}{ }_{\tau}= & -2 \varepsilon_{i} \omega_{i} b_{i}^{(2)}-\omega_{i}^{2} b_{i}^{(1)}-\sum_{j} \sum_{k} D_{i j k} b_{j}^{(1)} b_{k}^{(1)} \\
& -\sum_{j} \sum_{k} \sum_{l} E_{i j k l} b_{j}^{(1)} b_{k}^{(1)} b_{l}^{(1)}+p_{d} G_{i} \cos \omega \tau,(i, j, k, l=1,2,3, \ldots)
\end{aligned}
$$

where $b_{i}^{(1)}(\tau)$ and $b_{i}^{(2)}(\tau)$ correspond to the displacement and the velocity in the state space, respectively. In Eq. (6), new dissipation terms of linear damping are introduced. Damping ratio $\varepsilon_{i}$ corresponds to the $i$ - th mode of vibration.

Applying the harmonic balance method, the nonlinear periodic responses are calculated. The chaotic responses are numerically integrated by the Runge-Kutta-Gill method. To confirm whether random-like responses are the chaos, the Lyapunov exponents are calculated by the procedure proposed by Wolf et al. ${ }^{(5)}$ The Lyapunov dimensions are calculated from the Lyapunov exponents by the procedure by Kalpan\&Yorke ${ }^{(10)}$. When Lyapunov dimension converges to a constant value, one can estimate the number of vibration modes, which contribute to the chaos. Furthermore, principal components are calculated with the time responses at multiple positions of the beam.

\section{Results and discussion}

In the experiment, the non-dimensional spring coefficient $k$ of the axial spring is measured as $k=5.23 \times 10^{-3}$. The initial deflection is measured as:

$$
w_{0}=7.36 \zeta_{1}(\xi)-5.84 \zeta_{2}(\xi)+2.15 \zeta_{3}(\xi)-0.56 \zeta_{4}(\xi)+0.64 \zeta_{5}(\xi) \text {, }
$$

where $\zeta_{i}(\xi)$ is the mode shape function as explained in Eq. (5). The maximum amplitude of initial deflection is 2.1 times of the thickness of the beam. The static lateral load by gravity is $p_{s}=422$. The amplitude of periodic lateral excitation is $p_{d}=1120$. Nonlinear responses of the beam are detected at the position $\xi=0.7$. In the experiment, the measuring positions are selected as $\xi=0.06,0.25,0.50,0.70$ and 0.90 for the principal component analysis. In the analysis, the damping ratio $\varepsilon_{i},\left(i=1,2, \ldots, I_{\mathrm{c}}\right)$ is assumed as $\varepsilon_{i}=0.006$, where $I_{c}$ represents the maximum number of vibration modes. To approximate the static deflection and the dynamic response, five terms expansion of the mode shape function is used in Eq. (5). The time-step increment of the numerical integration is chosen as $1 / 240$ of the forcing period $\tau_{e}$. To avoid transient response due to computational initial conditions on the chaotic responses, time series after $3000 \tau_{e}$ are selected in the evaluation of the chaotic vibrations. Time responses at twenty positions on the beam are used for the principal component analysis.

\subsection{Linear natural frequencies and natural vibration modes of the beam}

Fig.4 shows the linear natural frequencies $\omega_{1}, \omega_{2}$ of the beam under the axial compressive force $-n_{x}$ by the spring. In the fig.4 (a), the ordinate indicates linear natural frequencies $\omega_{i}(i=1,2)$. The abscissa is the normalized axial compression $n_{x} / n_{c r}$ by the critical force $-n_{c r}$. The solid line represents the analytical result and the black circle denotes the experimental results with $\beta=0.4$. The broken line and white circle represent the analytical results and experimental results with $\beta=0$, respectively. The dashed dotted line denotes analytical results with $w_{0}=0, p_{s}=0$. In the fig.4 (b), the abscissa is the axial compressive displacement $-u_{0}$. The two-dot chain line represents the two conditions of the following experimental results.

For $\beta=0$ in the experiment, increasing the compressive force from $n_{x} / n_{c r}=0$, the frequencies decreases gradually. Increasing the axial force from $n_{x} / n_{c r}=0.75$ close to the 
critical force $n_{x} / n_{c r}=1$, the natural frequencies increase steeply owing to the post-buckled deflection of the beam with initial deflection. When the concentrated mass $\beta=0.4$ attached at $\xi_{1}=0.3$, the natural frequencies $\omega_{1}, \omega_{2}$ decrease. Although, for the $\beta=0.4$, the discrepancy appears between experimental and analytical results. The discrepancy is $3.7 \%$ $(\Delta \omega=1.4)$ in the second natural frequency $\omega_{2}$. The analytical results show the same behavior as the experimental results of the natural frequency.

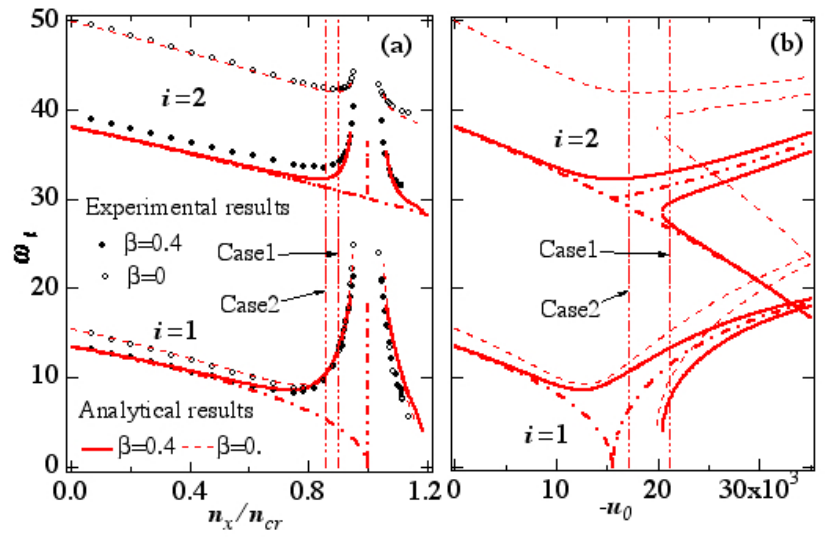

Fig. 4 Linear natural frequencies of the beam under axial compression $-n_{x} .-n_{c r}$ is a critical force of the buckling. Experimental results $\left(-n_{c r}=19.4\right)$. Analytical results $\left(-n_{c r}=20.2\right) ;-\cdot-w_{0}=0, p_{s}=0$. $-\cdots-\operatorname{Case} 1\left(n_{x} / n_{c r}=0.90,-u_{0}=21200\right), \operatorname{Case} 2\left(n_{x} / n_{c r}=0.86,-u_{0}=17200\right)$.
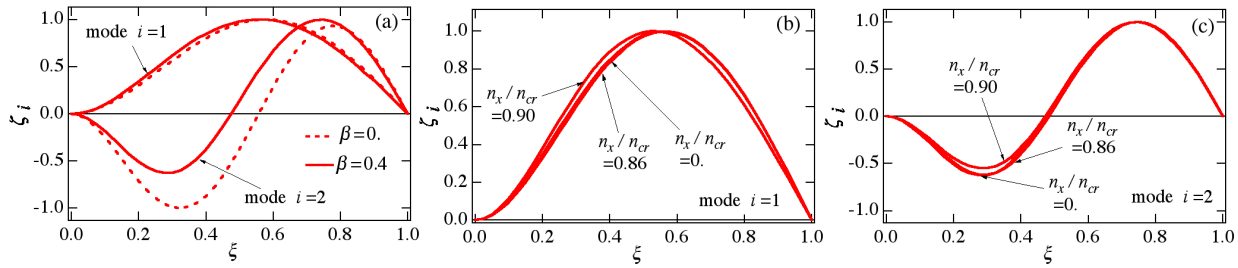

Fig. 5 Linear natural vibration modes of the beam with a concentrated mass under axial compression. Analytical results; (a): first and second modes with $n_{x} / n_{c r}=0,--\beta=0$. and $-\beta=0.4$,

(b): first mode with $\beta=0.4$, (c): second mode with $\beta=0.4$.

Fig.5 (a) shows the linear natural vibration modes for $\beta=0$ and $\beta=0.4$ in the analysis. For the lowest mode, the amplitude of the beam increases slightly around the position of the concentrated mass $\xi_{1}=0.3$. For the second mode, the amplitude decreases drastically at $\xi_{1}=0.3$. Fig.5 (b) and (c) represent the natural vibration modes for $\beta=0.4$. As the axial compression increases in the post-buckled state of the beam, the configuration of the lowest vibration mode is transformed from asymmetric to symmetric configuration, and the amplitude of the second vibration mode at $\xi_{1}=0.3$ decrease.

\subsection{Restoring force of the beam}

Fig. 6 shows the static lateral deflection $w$ of the beam under the concentrated force $q_{s}$ loaded on the center of the beam. The notation of non-dimensional deflection is introduced as $w=W / h$ for the comparison of experimental and analytical results. The deflection is measured at the position $\xi=0.7$. The origin of deflection is selected at the static equilibrium position of the beam under the axial compressive force and the gravity. For $n_{x} / n_{c r}=0.90$ (Case1), the characteristics of restoring force of the beam show the type of a softening-and-hardening spring including a negative gradient. In this case, the characteristics have two static equilibrium points. Decreasing the axial compression to $n_{x} / n_{c r}=0.86$ (Case2), the negative gradient decrease as the decrease of curvature caused by the axial compression. 


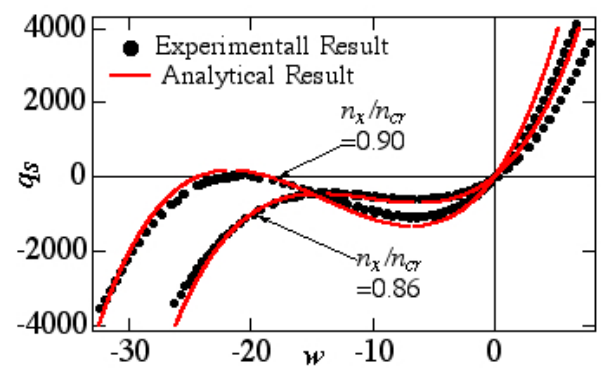

Fig. 6 Characteristics of the restoring force; concentrated load $q_{s}$ at $\xi_{2}=0.5$.

\subsection{Frequency response curve of the beam}

Nonlinear response curves of the beam are shown in Fig. 7. The ordinate $w_{\mathrm{rms}}$ indicates the root mean square value of the dynamic response at the position $\xi=0.7$ of the beam. The abscissa $\omega$ denotes the non-dimensional exciting frequency. Notation $(i: j)$ indicates the type of resonance response, in which the integer $i$ is a generated mode of vibration, while the index $j$ denotes a type of resonance. For example, $i=1$ represents the principal resonance, while $j=1 / 2$ means the sub-harmonic resonance of $1 / 2$ order. The chaotic response is shown by the notation $\mathrm{C}(i: j)$, in which the notation $(i: j)$ means the predominant type of resonance in the chaotic response. The black circles indicate experimental results, and the arrows show the jump phenomena where a response transits to another response. The solid lines indicate nonlinear periodic response in the analysis, and the dotted lines shows the unstable periodic responses. The dashed and dotted line indicates nonlinear free vibration corresponding to the lowest mode of vibration. Natural frequencies of the experimental result are indicated by the inverse triangles on the abscissa.

For the $n_{x} / n_{c r}=0.90$ (Case1), the chaotic responses dominated dynamic snap-through are generated within wide frequency range from the non-resonant response with the jump phenomenon. As will be shown later in Fig.11, the maximum Lyapunov exponents of the chaotic response have positive values, then the response is confirmed as the chaos. The chaotic response $C(1: 2 / 3)$ is dominated predominantly by the ultra-sub-harmonic resonance response of $2 / 3$ order with the lowest mode of vibration. For the $n_{x} / n_{c r}=0.86$ (Case2), the chaotic response $\mathrm{C}(1: 3 / 8 ; 2: 2)$ appears within a narrow frequency range corresponding to the ultra-sub-harmonic resonance response of $3 / 8$ order with the lowest mode of vibration and the super-harmonic resonance response (2:2) of the second mode of vibration.
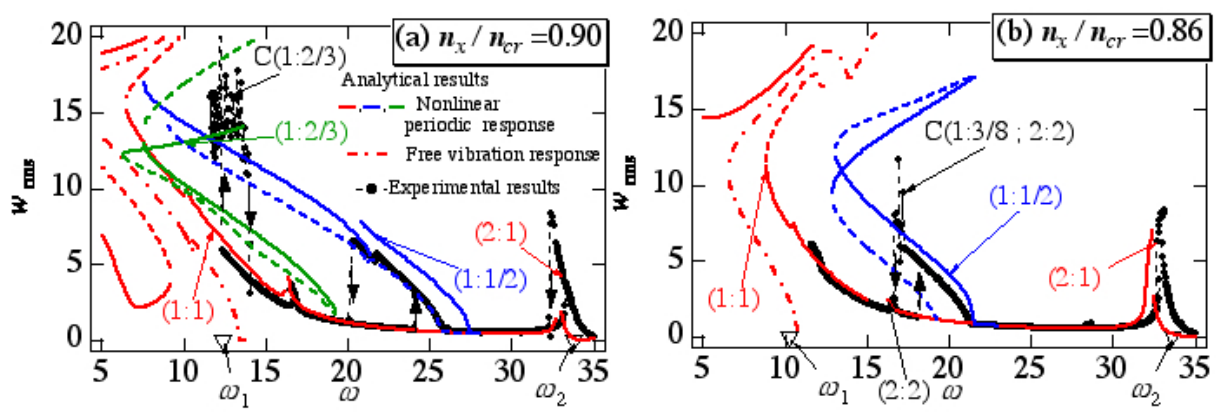

Fig. 7 Frequency response curve of the beam under axial compression. $p_{d}=1120, \xi=0.7$.

$\nabla$ experimental results of natural frequencies $\omega_{1}, \omega_{2}$.

\subsection{Time histories, Poincaré projections and Fourier spectra of the chaos}

The time histories are examined by the Fourier spectra. In the chaotic response $\mathrm{C}(1: 2 / 3)$, the results at the exciting frequencies $\omega=12.15$ in the experiment and $\omega=13.50$ in the analysis are shown in Fig.8. In the left-side figure, the time histories of the non-dimensional deflection $w$ are shown by the time scale of excitation period $\tau_{e}$. The 
Fourier spectra of these responses are presented in the right-hand-side figures. In the figure, the ordinate stands for the Fourier amplitude $A$ of which level is scaled with decibel, while the abscissa indicates the non-dimensional frequency of spectrum $\omega_{s p}$ with logarithmic scale. In the time histories, large amplitude chaotic responses of the beam can be observed transits irregularly between the two stable equilibrium points, $w=0$ and $w=-25$, involving dynamic snap-trough. Fourier spectra of the chaotic response has lump spectrum around the frequency two-third of the exciting frequency $\omega$. Therefore, the chaotic response $\mathrm{C}(1: 2 / 3)$ is generated from the predominant response of the ultra-sub-harmonic resonance of $2 / 3$ order.
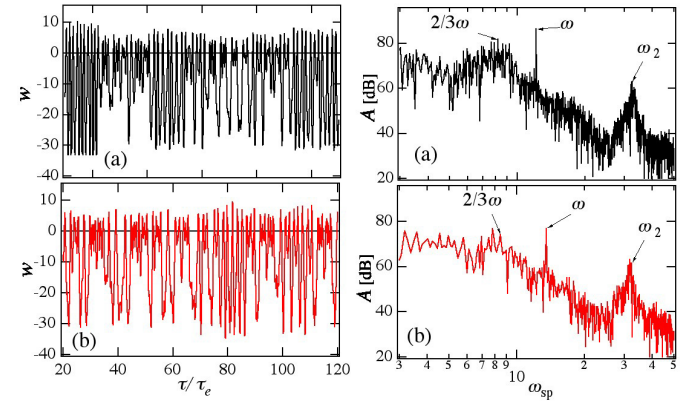

Fig. 8 Time progress and Fourier spectra of the responses of the beam $\mathrm{C}(1: 2 / 3)$, result $\omega=12.15$, (b) Analytical results $\omega=13.50$.

(a) Experimental

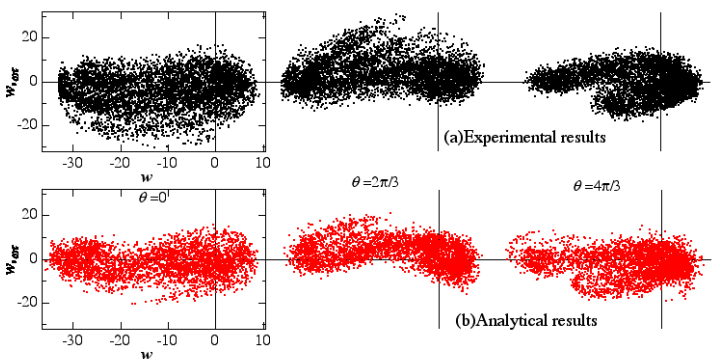

Fig. 9 Poincaré projection in each phase delay $\theta, \mathrm{C}(1: 2 / 3)$, (a)Experimental results $\omega=12.15$, (b)Analytical results $\omega=13.50$.
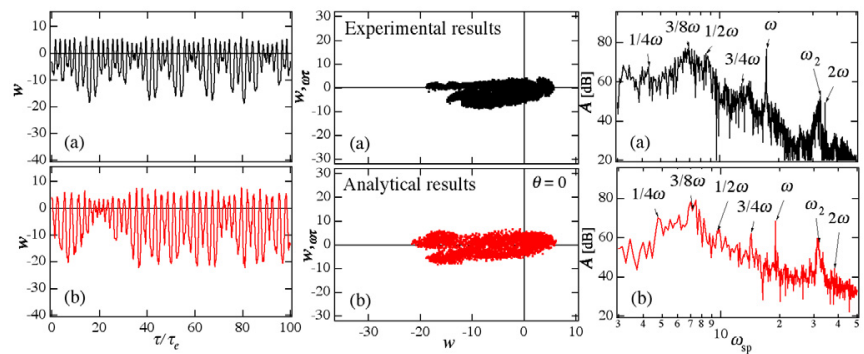

Fig. 10 Time progress, Poincré projection and Fourier spectra of the responses of the beam $\mathrm{C}(1: 3 / 8 ; 2: 2)$, (a) Experimental result $\omega=17.32$, (b) Analytical results $\omega=19.07$.

Fig.9 shows the variation of the Poincare projections for the chaotic response of $\mathrm{C}(1: 2 / 3)$ changing the phase delay $\theta$ with the increment of $2 \pi / 3$ radians. The deflection $w$ and the velocity $w_{\omega \tau}$ at the position $\xi=0.7$ of the beam are recorded in every period of the excitation. In the projections, 6000 points are plotted on the phase plane. The Poincaré projections of the chaotic response of the type $\mathrm{C}(1: 2 / 3)$ spread out to a scattered figure. A fractal pattern is clearly constructed in this Poincaré projection. As the phase angle is shifted, the projection rotates clockwise accompanied by the folding-and-stretching in the fractal figure. Both projections of the experiment and the analysis have similar figures to each other. Fig. 10 shows the time histories, the corresponding Fourier spectra and the Poincare projections of the responses of $\mathrm{C}(1: 3 / 8 ; 2: 2)$. The time histories show small 
irregular amplitude modulation. The corresponding Fourier spectra show the distinct sub-harmonic components of orders $i / 8(i=2,3,4, \ldots)$ and second natural frequency $\omega_{2}$. The second natural frequency $\omega_{2}$ has a condition of $\omega_{2} \approx 2 \omega$. The related Poincaré projections differ qualitatively compared with the chaos $\mathrm{C}(1: 2 / 3)$ involving dynamic snap-trough.

\subsection{Maximum Lyapunov exponent and Lyapunov dimension}

Fig.11(a) shows experimental result of the maximum Lyapunov exponents $\lambda_{\max }$ related to the embedding dimension $e$ of the chaotic response $\mathrm{C}(1: 2 / 3)$. As the embedding dimension $e$ increase to $e=6$, the maximum Lyapunov exponent $\lambda_{\max }$ converges to $\lambda_{\max }=1.44$. Moreover, the number of the predominant modes of vibration that contribute the chaos is counted as three. Fig.11(b) shows the Lyapunv dimensions $d_{L}$ by changing the assumed number of vibration modes $I_{\mathrm{c}}$ in the analysis. When the assumed mode $I_{\mathrm{c}}$ increases to $I_{\mathrm{c}}=3$, the Lyapunov dimension $d_{L}$ converges to the dimension $d_{L}=4.9$, and the corresponding maximum Lyapunov exponent is $\lambda_{\max }=1.39$. Consequently, the number of vibration modes contribute to the chaos is three. In the chaotic response $C(1: 2 / 3)$, the maximum Lyapunov exponent $\lambda_{\max }=1.39$ in the analysis agrees fairly well with the exponent $\lambda_{\max }=1.44$ in the experiment. Moreover, the number of vibration modes coincides with the experimental and analytical results. Fig.12 (a) shows experimental result of the maximum Lyapunov exponents $\lambda_{\max }$ of the chaotic response $\mathrm{C}(1: 3 / 8 ; 2: 2)$. As the embedding dimension $e$ increase to $e=4$ to $e=6$, the maximum Lyapunov exponent $\lambda_{\max }$ converges to $\lambda_{\max }=0.75$. The number of the predominant modes of vibration that contribute the chaos is counted as three. Fig.12 (b) shows the Lyapunv dimensions $d_{L}$ in the analysis. When the assumed mode $I_{\mathrm{c}}$ increases to $I_{\mathrm{c}}=2$ to $I_{\mathrm{c}}=3$, the Lyapunov dimension $d_{L}$ converges to the dimension $d_{L}=3.5$, and the corresponding maximum Lyapunov exponent is $\lambda_{\max }=0.76$. Consequently, the number of vibration modes contribute to the chaos is two or three. In the chaotic response $C(1: 3 / 8 ; 2: 2)$, the maximum Lyapunov exponent $\lambda_{\max }=0.76$ in the analysis agrees fairly well with the exponent $\lambda_{\max }=0.75$ in the experiment.

\subsection{Contribution of vibration mode of the chaotic responses}

The analytical procedure with the multiple-degree-of-freedom system enables the inspection of the chaotic responses related to the individual natural modes of vibration. Fig. 13 shows the Poincare projections of the chaotic response $\mathrm{C}(1: 2 / 3)$, in which the projection for each vibration mode is displayed. In the figure, the Poincare projection of $b_{i}$ and $b_{i}, \omega \tau\left(i=1,2, \ldots, I_{\mathrm{c}}\right)$ corresponds to the $i$-th mode of vibration. The notation $b_{i}$ represents the displacement at the position of maximum amplitude of the $i-$ th mode. The Poincaré projection of $w$ and velocity $w,{ }_{\omega \tau}$ is defined as an overall projection.

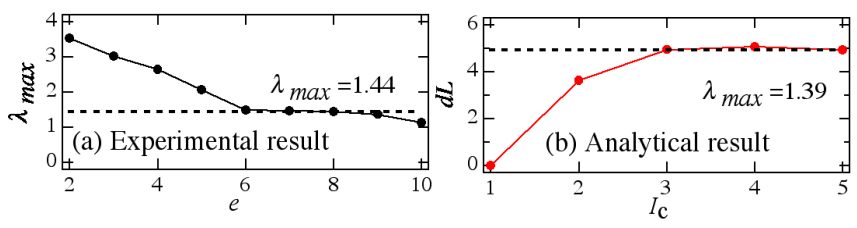

Fig. 11 Maximum Lyapunov exponent related to embedding dimension and Lyapunov dimension. $\mathrm{C}(1: 2 / 3)$; (a)Experimental result $\omega=12.15$, (b)Analytical result $\omega=13.50$
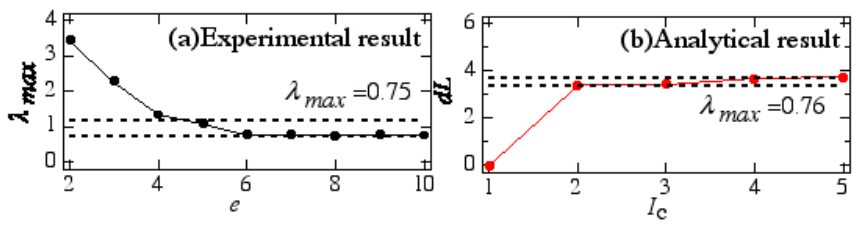

Fig. 12 Maximum Lyapunov exponent related to embedding dimension and Lyapunov dimension. $\mathrm{C}(1: 3 / 8) ;(\mathrm{a})$ Experimental result $\omega=17.32$, (b)Analytical result $\omega=19.07$. 


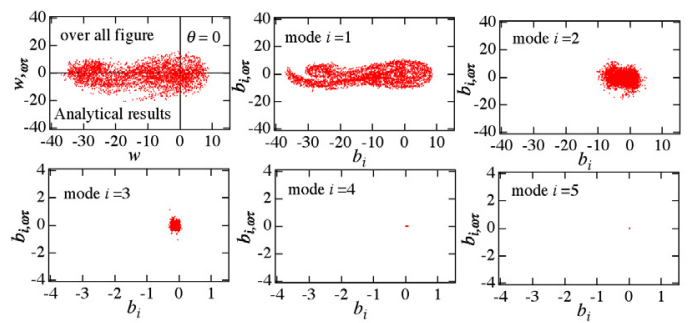

Fig. 13 Poincare projection in each mode. Analytical results; $\mathrm{C}(1: 2 / 3): \omega=13.50$.

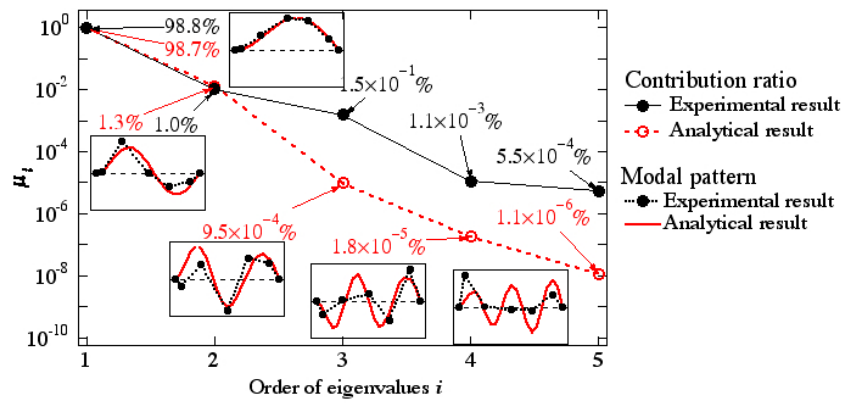

Fig. 14 Principal components in the chaos. Experimental result $\omega=12.15$, Analytical results $\omega=13.50$

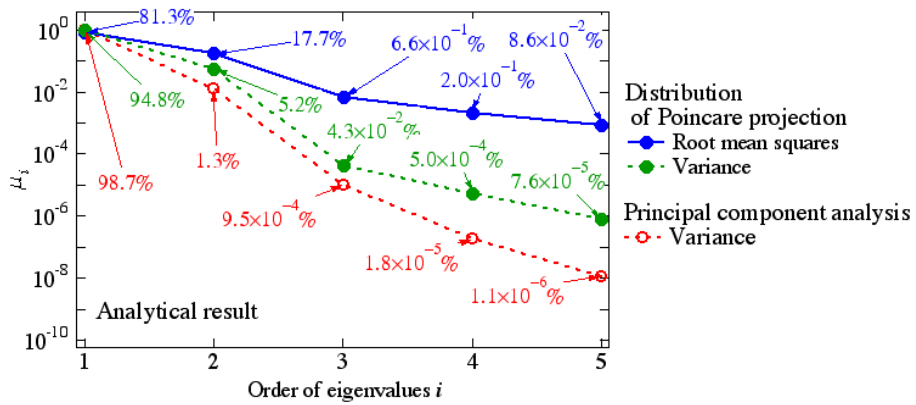

Fig. 15 Difference of the contribution order of the chaos $C(1: 2 / 3)$ resulted from evaluation values.

From the figures, the attractor of the lowest mode of vibration has a similar figure to the overall projection. The lowest mode of vibration has dominant contribution to the chaotic response with $81.3 \%$ of the root-mean-square value calculated from the distribution of the displacement $b_{i}$. For higher modes of vibration, the chaotic response $\mathrm{C}(1: 2 / 3)$ involves the second mode and third mode with $17.7 \%$ and $0.7 \%$, respectively.

Applying the principal component analysis, contribution ratio and related modal pattern are calculated. Fig. 14 shows the experimental and analytical results of the chaotic response $\mathrm{C}(1: 2 / 3)$. The ordinate indicates the contribution ratio $\mu_{i}$ of each principal component, while the abscissa is the order of eigenvalue $i$. Modal patterns are also illustrated in the figure. In the experiment, the largest principal component, which corresponds to the lowest vibration mode, prevails the contribution ratio of $98.8 \%$. The contribution ratios of the second and third modes are $1.0 \%$ and $0.15 \%$, respectively. In the analysis, the lowest, the second and third modes are $98.7 \%, 1.3 \%$ and $9.5 \times 10^{-4} \%$, respectively. The contribution ratios of the lowest and the second modes agree well in the experiment and analysis.

Comparing to the results of the maximum Lyapounov exponent, the modal patterns of which contribution ratio is more than $10^{-6}$ order, mainly contribute to the chaos.

Fig. 15 shows the contribution ratios of the chaos $\mathrm{C}(1: 2 / 3)$ evaluated by variance and root-mean-square value of the displacement $b_{i}\left(i=1,2, \ldots, I_{\mathrm{c}}\right)$ of the Poincare projection and the contribution ratio calculated by the principal component analysis. The results of the principal component analysis correspond well to the contribution ratio calculated by the variance of the Poincaré projection, because both contribution ratios are based on the 
variance. The contribution ratio estimated by the root-mean-squares value of the displacement of the Poincare projection is larger than the others.

\section{Conclusions}

Experiments and analysis have been carried out on the chaotic vibrations of a clampedsupported beam with a concentrated mass in several conditions of the axial compression. The main results are summarized as follows:

(1) For the chaotic vibration of the ultra-sub-harmonic resonance responses of $2 / 3$ order dominated by dynamic snap-through, Poincaré projection and maximum Lyapunov exponent in the experiment and analysis agree fairly well in detail. The maximum Lyapunov exponent is the order around $\lambda_{\max }=1.4$. By the saturated maximum Lyapunov exponent to the embedding dimension and Lyapunov dimension, the number of the vibration modes contributed to the chaos is counted as three.

(2) The chaotic vibration accompanied with the internal resonance induced within narrow frequency range. The maximum Lyapunov exponent is the order around $\lambda_{\max }=0.8$. The number of the vibration modes contributed to the chaos is counted as two or three.

(3) The contribution ratio calculated by the principal component analysis indicate smaller value than the contribution ratio estimated by the distribution of displacement of the beam, for the modes with relatively small contribution.

\section{References}

(1) Nagai, K., Yamaguchi, T., Chaotic Vibrations of a Post-buckled Beam Carrying a Concentrated Mass (1st Report, Experiment), Transactions of the Japan Society of Mechanical Engineers, Series C, Vol. 60, No. 579(1994), pp.3733-3740.

(2) Yamaguchi, T., Nagai, K., Chaotic Vibrations of a Post-buckled Beam Carrying a Concentrated Mass (2nd Report, Theoretical Analysis), Transactions of the Japan Society of Mechanical Engineers, Series C, Vol. 60, No. 579(1994), pp.3741-3748.

(3) Nagai, K., Maruyama, S., Sakaimoto, K. and Yamaguchi, T., Experiments on Chaotic Vibrations of a Post-buckled Beam with an Axial Elastic Constraint, Journal of Sound and Vibration, Vol. 304(2007), pp.541-555.

(4) Yanagisawa, D., Nagai, K. and Maruyama, S., Effects of Axial Compressive Load on Chaotic Vibrations of a Clamped-Supported Beam, Transactions of the Japan Society of Mechanical Engineers, Series C, article in press.

(5) Wolf, A., Swift, JB., Swinney, H.L. and Vastano, J.A., Determing Lyapunov Exponents from a Time Series, physica, D, Vol. 16, (1985), pp.285-317.

(6) Takens, F., Detecting Strange Attractors in Turbulence, Lectures Notes in Mathematics, Vol. 898(1981), pp.366-381.

(7) Loéve, M.M., Probability Theory, Von Nostrand, Princeton NJ, 1955.

(8) Yamaguchi, T., Nagai, K. and Maruyama, S., Identification of Spatial Modes in Chaotic Vibration Involving Dynamic Snap-through Using KL Method (Numerical Study for a Uniform Beam including Geometric Nonlinearity in a Multiple-degree-of-freedom System), Transactions of the Japan Society of Mechanical Engineers, Series C, Vol. 69, No. 687(2003), pp.2973-2942.

(9) Nagai, K., Nagaya, K., Takeda, S. and Arai, N., A Free Vibration of Beams Carrying a Concentrated Mass under Distributed Axial Forces, Transactions of the Japan Society of Mechanical Engineers, Series C, Vol. 54, No. 497(1988), pp.39-46.

(10) Kalpan, J., Yorke, J., The Lyapunov Dimension of Strange Attractors, Journal of Differential Equations, 49(1983), pp.185-207. 